Editorial

\title{
Acknowledgement to Reviewers of Econometrics in 2017
}

Econometrics Editorial Office

MDPI AG, St. Alban-Anlage 66, 4052 Basel, Switzerland

Published: 10 January 2018

Peer review is an essential part in the publication process, ensuring that Econometrics maintains high quality standards for its published papers. In 2017, a total of 47 papers were published in the journal. Thanks to the cooperation of our reviewers, the median time to first decision was 55 days and the median time to publication was 141 days. The editors would like to express their sincere gratitude to the following reviewers for their time and dedication in 2017:

\author{
Abadie, Alberto \\ Alexeev, Vitali \\ Amisano, Gianni \\ Ashley, Richard \\ Assenmacher, Katrin \\ Audrino, Francesco \\ Bacchiocchi, Emanuele \\ Bartolucci, Francesco \\ Bazen, Stephen \\ Berghaus, Betina \\ Biewen, Martin \\ Billio, Monica \\ Binder, Carola \\ Bolotova, Yuliya \\ Bonaccolto, Giovanni \\ Borovkova, Svetlana \\ Bosquet, Clement \\ Boswijk, Peter \\ Boudt, Kris \\ Bücher, Axel \\ Camacho, Maximo \\ Caporale, Guglielmo \\ Caporale, Guglielmo Maria \\ Caporin, Massimiliano \\ Casas, Isabel \\ Castle, Jennifer \\ Chao, John \\ Chaussé, Pierre \\ Chavleishvili, Sulkhan \\ Cook, C Justin \\ Creel, Michael \\ Danan, Eric \\ Del Barrio Castro, Tomás \\ Drydakis, Nick
}

Égert, Balázs

Elliott, Graham

Fanelli, Luca

Franchi, Massimo

Franses, Philip Hans

Gallant, A. Ronald

Garderen, Kees Jan Van

Genest, Christian

Giorgi, Giovanni Maria

Golez, Benjamin

Golosnoy, Vasyl

Górecki, Jan

Granziera, Eleonora

Gulasekaran, Rajaguru

Hagemann, Andreas

Hannig, Jan

Hansen, Peter R.

Harding, Matthew

Harvey, Andrew

Hassler, Uwe

Heravi, Saeed

Herbst, Ed

Hetland, Andreas

Hidalgo, Javier

Hjalmarsson, Erik

Hoga, Yannick

Inoue, Atsushi

Jales, Hugo

Jansson, Michael

Jasiak, Joann

Johansen, Søren

Khashanah, Khaldoun

Kim, Min Seong

Kim, Aaron 
Koopman, Siem Jan

Kosfeld, Reinhold

Kovchegov, Yevgeniy

Krisztin, Tamas

Kuffner, Todd

Kurita, Takamitsu

Lamarche, Carlos

Lee, Yoonseok

Lee, Young $\mathrm{H}$.

LeSage, James $P$.

Liu, Laura

Liu, Long

Longhi, Simonetta

Lubrano, Michel

Lucchetti, Riicardo (Jack)

Lye, Jenny

Magdalou, Brice

Maneesoonthorn, Worapree Ole

Mantalos, Panagiotis

Marcucci, Juri

Maugis, Pierre-André

McCloskey, Adam

Mcmillan, David

Meinecke, Juergen

Moulton, Jeremy Grant

Nielsen, Bent

Nielsen, Heino Bohn

Ning, Cathy

Noack, Andreas

Nolte, Ingmar

Okui, Ryo

Ordóñez, Javier

Panopoulou, Ekaterini

Pappadà, Roberta

Park, Joon Y.

Paruolo, Paolo

Patton, Andrew J.
Pearl, Judea

Pedersen, Rasmus Søndergaard

Peters, Gareth W.

Pfaffermayr, Michael

Pinto, Cristine

Raïssi, Hamdi

Reboredo, Juan

Rémillard, Bruno

Schluter, Christian

Sickles, Robin C.

So, Mike Ka Pui

Sordo, Miguel A.

Spanos, Aris

Stark, Tom

Stillwagon, Josh

Swanson, Norman R

Tabor, Morten Nyboe

Tarsitano, Agostino

Tchatoka, Firmin Doko

Vahey, Shaun

Victoria-Feser, Maria-Pia

Wagner, Helga

Wagner, Martin

Walker, Patrick

Westerlund, Joakim

Wichitaksorn, Nuttanan

Wied, Dominik

Windmeijer, Frank

$\mathrm{Xu}$, Wen

Yalonetzky, Gaston

Yan, Wanfeng

Yang, Xiye

Yao, Wenying

Zelli, Roberto

Zhou, Qiankun

(C) 2018 by the author; licensee MDPI, Basel, Switzerland. This article is an open access article distributed under the terms and conditions of the Creative Commons Attribution (CC-BY) license (http://creativecommons.org/licenses/by/4.0/). 